PPFL -2185

UC2O-G

\title{
$1-19388$ (2)
}

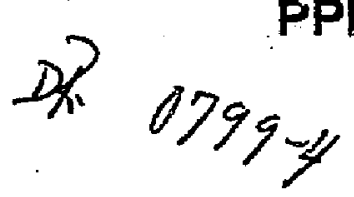

\section{PPPL-2.185}
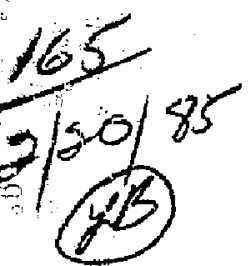

THEORY OF SEMICOLLISIONAL

DRIFT-INTERCHANGE MOLES IN CYLINDRICAL PLASMAS

By

T.S. Hahm and L. Chen

JANUARFं 1985

\section{PLASMA \\ PHYSICS \\ LABORATORT}

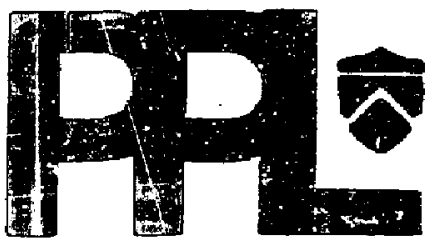

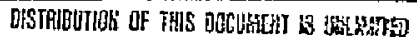

PRINCETON UNIIVERITY PRINCETON, NEW J2RSEY

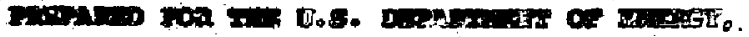

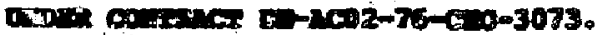


THEORY OF SAMICOLUIS IONAL

DRIFT-INTERCHANGE MODES IN CYLINDRICAL PLASMAS

\author{
PPPL- -2185 \\ by \\ DEBS 007222 \\ T. S. $\mathrm{Kahm}^{+}$and Liu Chen \\ Plasma Physics Laboratory, Princeton University \\ Princeton, New Jersey 08544
}

\begin{abstract}
Resistive interchange instabilities in cylindrical plasmas are studied. including the effects of electron dlamagnetic drift, perpendicular resistivity, and plasma compression. The analyses are pertinent to the gemicollisional tegime where the effective fon gyro-radiug is larger than the resistive layer width. Both analytical and nwerical results show that the modes can be completely stabilized by the perpendicular plasma transport. Ion sound effects, meanwhle, are found to be negligible in the semicollisional regine.
\end{abstract}

\title{
DISCLAIMER
}

This report was prepared ar an account of worl

Government. Neither the an account of work sponsored by and

employces, makes any warramty, States Government nor any agency of the United States process dist accuracy, completeness, or or implied, ot assumes ancy thereot, nor any of their process disclosed, or represents that, or usefulness of any infermatiogal liability or responsjmenuf act in to any specific commerciol use would not infringe privately owartus, product, or menufacturer, or otherwise does not necesset, process, or service by trade nod rights. Referand opinion favoring by the United States constitute or imply its endorse, trademark, United Stares of authors expressed herejn Government or apy agescy thersement, recominted Stares Government or any agency the not necessarily stuze of thereol. The views

'Present address: Inst1tute for Fusion Studies, University of Thxas, Austin, Texas 78712 


\section{INTRODUCTION}

The simple resistive magnetohydroiynamic (Mmo) theory in a sheared slab model with curvatire sfmulated by gravity predicts an unstable resistive interchange mode with a grosth rate proportional to the cubic root of the resistivity.' In a cylindrical model with uncavorable curvature and pressure gradlent, plagma compresgion gives a stabiliaing effect although it does not stabllize all the modes completely. ${ }^{2}$

These rrevtous models, however, do not provide adequate descriptions for plasmas encountered in present or future machines. In particular, electron diamagnetic drift frequency $\omega_{\star_{e}}$ can be greater than or comparable to the growth rate $\gamma$ of resistive interchange mode. For example, 2T-40M parameters ${ }^{3}$ give $\omega_{*} / \gamma \approx 3 \mathrm{~m}^{1 / 3}$, where $\pi$ is the poloidal mode number. Itis electron diamaqnetic drift effect hs been treated in the low- $\beta$; $\beta$ plasma pressure/magnetic pressure) linit where pressure is assumed to be convected (i.e., plasma compresstonal effects are missing in this model) and found to reduce the qrowth rate of the mode greatly although the mode remains unstable even for arbitrarily lafge $\omega_{\omega_{e}}{ }^{4-6}$

Another effect the usul restgtive HHD theories have neglected is the fact that the ion gyro-radilis at electron temperature $I_{g}=c_{s} / Q_{i}$ where $c_{s}$ is the lon sound speed $\left(\mathrm{T}_{\mathrm{e}} / \mathrm{H}_{i}\right)^{1 / 2}$ and $\Omega_{i}$ is the ion cyclotren frequencyl is often larger than the nominal resistive layer width $L_{F}=\left(\rho \eta^{2} a^{2} / n^{2} B_{\theta}{ }^{2} q^{2}\right)^{1 / 6}$. Agal, for ZT-4OM parameters, ${ }^{3}$ we have $\rho_{S} / L_{x}=6 \mathrm{~m}^{1 / 3}$. This is the so-called semicollisional regime. 7

Although there have been extensive studies on drift-tearing modes in the semicollisional regime, ${ }^{3,8}$ relatively little attention ba been pald to driftinterchange modes. Recently, F1nn et al. 9 (hereafter referred to as $\mathrm{MA}$ ) stuiled the stability of drift-interchange mudes in cylindrical geometry 
tneluding the effects of $\omega_{w_{e}} 2 s$ well as flnite plasma compression and ion polarization drift. Their equations, in fact, can be shown to be valla not only in the collisional reqime but also in the semicollisional regime. They found numerically that the drift-interchange node can be conpletely stabilized for a moderate value of $\omega_{w_{e}}$ by incluaing plasma compression, and concluded that the principal stabilizing influenceg are plasma conpression and coupling to out-going drift waves.

We have done extensive analytical and numerical studies on driftinterchange modes using the same equations FMA used. Specifically, an analytical dispersion relation has been derived via asymptotic matching and agrees excellently with rirect numerical solutions. We find, furthermore, that the principal stabilizing effect is due to the perpenticular plasma transport as well as plasma compression instead of coupling to drift waves as indicated by FMA. In fact, we find that while parallel compression of plasma (i.e., ion sourd effects which stabilize drift waves) reduces the growth rates of the modes in the collisional regime, it has a negligible effect in the semicollisional egime.

In sec. 2, we present the efgernode equations and explain the physical meanings of various terms. An analytical dispersion relation for the semicolisional drift-interchange mode is then derived in sec. 3. Properties of that dispersion relation are discussed in sec. 4. Section 5 contains direct numerical solutions which are essentially the same as those of FMa and are slown to agree excellently with the analyttcal dispersion relation. Final conclusions and discussions are given in sec. 6. 


\section{BASIC EQUATIONS}

In cylindrical geonetry, the equations for resigtive modes inciuding the additional effects of electron diamagnetle drifts and ion polarization drift (semicollysional effects) can be derived by the procedure outlined in fMA and WII not be repeated here. The equations in the normalized variables are given by

$$
\begin{aligned}
& \frac{\partial^{2}}{\partial x^{2}} \Xi=\frac{x}{\rho^{2}} \frac{\partial^{2}}{\partial x^{2}} \Psi-\frac{1}{Q^{2}} T \\
& \frac{\partial^{2}}{\partial x^{2}} \Psi=Q(\Psi+x E)+i \rho_{t} \Psi+\frac{2 i g X}{s} T, \\
& \frac{\pi_{\perp}}{\pi_{\partial}} \frac{\partial^{2}}{\partial x^{2}} T=Q\left(1+\frac{x^{2}}{\rho^{2}}+\frac{2}{\Gamma B}\right) T+\rho\left(g-D-\frac{2 D}{\Gamma \beta}\right) \Xi+\frac{D}{Q} x \Psi-2 i Q^{2} g \frac{\partial^{2}}{\partial x^{2}} \Xi
\end{aligned}
$$

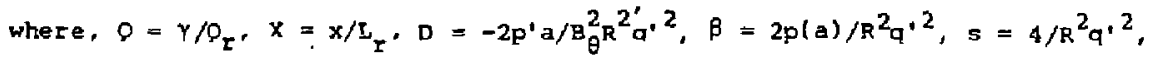

$$
\begin{aligned}
& \Psi=\frac{-i a \tilde{B}_{r}}{{ }^{R B} \theta^{q^{\prime} L_{r}}} \text {, } \\
& T=\frac{-2 \mathrm{Ba} \tilde{B}_{\|}}{R^{2} q^{, 2} B_{\theta}^{2}}=-\frac{2 a \tilde{p}}{R^{2} q^{\prime 2}{ }^{2}{ }_{\theta}^{2}} \text {. } \\
& L_{x}=\left(\frac{\rho \eta^{2} a^{2}}{\left.n^{2} B_{\theta^{2}}^{2}\right)^{2}}\right)^{1 / 2} \\
& \rho_{r}=\left(\frac{\eta_{n^{2} B_{\theta}^{2} q^{2}}^{2}}{\rho_{a}^{2}}\right)^{1 / 3} \\
& q=\frac{\left[a v_{A} / \pi\right)^{1 / 3}\left|n q^{\prime} a\right|^{1 / 3} v_{A}}{\left|k g^{\prime}\right| a_{A} a}
\end{aligned}
$$

and the rest of the notationg are standard. In deriving Eqs. (1) - (3), one ha asgumed $T_{i} \ll T_{e}, d\left(p_{i} / \rho \Gamma\right) / d t=0$, and eiectrons are 1sothermal. We have 
differentiated $\eta_{\perp}$ and $n_{1}$ in 29 . (3) in order to isolate the effects of the perpendicular reaigtivity.

Now, we explain the physical meanings of each equation. Squation $(1)$ is the equation of motion in the radial direction. The first term on the righthand side (RHS) is the fleld line bending term and the second term is the pressure gradient times curvature (1.e., the interchange instability driving term): while the left-hand side (Lus) describes the inertia. Equation (2) is the parallel Ohm's law. The first term on the RHS is the parallel electric fieln, the second term comes fruin the gradient of the equilibriun electron pressure along the magnetic field til ted by perturbations, and the last tem describes the gradient of the perturbed electron pressure along the equilibrium maqnetic field direction. Meanwhile, the LHS is the resistivity times parallel current. (Note that resistivity is absorbed into normalized variables.) The last equation, 9 . (3), is the pressure evolution equation. QT and $Q(s$ - D) $\Xi$ represent the temporal and convective changes of the pressure, respectively. $(2 \rho / \Gamma \beta)(T-D E)$ comes from the divergence of the perpendicular aisplacement. $\left(\pi_{\perp} / \eta_{\|}\right)\left(\partial^{2} \mathrm{~T} / \partial \mathrm{x}^{2}\right)$ represents the effect of plasma diffusion due to the perpendicular resigtivity. $x^{2} \mathrm{~T} / Q$ and $\mathrm{Dx} \Psi / Q$ are ion sound termg. The last term in 8 , (3) is the lon polarization drift term which is neglected in the collisional regire. This tern represents the demicollisional (finite $P_{3}$ ) effect. In the low- $\beta$ limit B. $(3)$ reduces to $T-D E=0$. In this limit, not only compresgional effects in both the parallel and the perpendicular directions (including ion polarization drift) but also perpendicular resistivity do not appear in the equations. Or the other hand, the full sixth order system of Eqs. (1), (2), and (3) is mard to treat analytically. This sygtem of equations can be reduced to a fourth order system by fourlec transformation. He present those fourier transformed 
equations in Sec. 5 where we discuss the numerical results. If weglect the Ion sound texms in Eq. (3), we can further reduce the order of the sygtem to second order. The second order equation we obtain is

$$
\begin{aligned}
& \frac{d}{d \theta} \frac{1+\theta^{2}}{1+\left[i \lambda /\left(\omega-\omega_{\star}\right)\right]\left(1+\theta^{2}\right)^{\frac{d}{d \theta}} \tilde{\phi}} \\
& +\left(\frac{\omega-\omega}{\omega-(1-\varepsilon) \omega_{*}}\right) \frac{(1-\varepsilon) D+\omega\left(\omega-\varepsilon \omega_{*}\right)\left(t+\theta^{2}\right)+\left(i \lambda_{\perp} \Gamma \beta / 2\right) \omega\left(1+\theta^{2}\right)^{2}}{1+\left\{\left(\varepsilon \omega \omega_{*}^{2} / D \cdot i \lambda_{1} \Gamma \beta / 2\right] /\left[\omega-(1-\varepsilon) \omega_{*}\right]\right\}\left(1+\theta^{2}\right)} \theta^{2}=0 ;
\end{aligned}
$$

where we have used [although $k_{\theta}^{2}$ has besn neglected in Eqs. $\left.(1)-(3)\right]_{1}^{2}=-$ $k_{\theta}^{2}+d^{2} / d r^{2}$ to remove the singularity at $\theta=0$. Fquation (4) is writcen in terms of variables adopted by connor et al. 10, $i$.e..,

$$
\tilde{\phi}=(2 \pi)^{-1 / 2} \int_{-\infty}^{\infty} d r \phi(r) \exp \left(-i k_{x} r\right)
$$

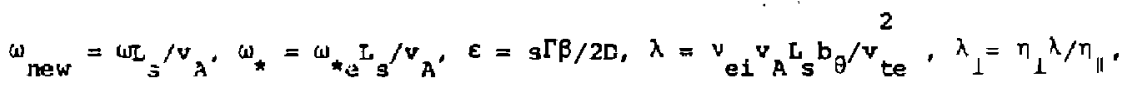
$\theta=k_{x} / k_{\theta}$, and $I_{g}$ is the shear length. Here, $\phi$, which is the nonadiabatic part of the electron density response, ${ }^{1 T}$ is related to the radial displacement $\xi_{\text {r }}$ by

$$
\phi=\frac{1+\left\{\left(\varepsilon \omega \omega_{*}^{2} / D+i \lambda_{\perp} \Gamma \beta / 2\right) /\left[\omega-(1-\varepsilon) \omega_{*}\right]\right\}\left(1+\theta^{2}\right)}{1+\left(i \lambda_{\perp} \Gamma \beta / 2 \omega\right)\left(1+\theta^{2}\right)} \xi_{\Sigma} .
$$

Eutation (4) is the elgenmode equation upon which our analytical theory is bassd. It will be ghown ir Sec. 4 that ion sound contribution 1s indeed negilgtble in the semicollisional regime of inter st here. 


\section{DERIVATION OF DISPERSION RELATION}

In this section, we derive a dispersion relation for the semicollisional drift-interchange modes. Flrgt, we neglect the perpendicular reglgtivity, but will include it as a perturbation in the next section. Neglecting the perpenaicular resistivity, Eq. (4) becomes, noting $b_{\theta}=E \omega_{*}^{2} / D_{\text {, }}$

$$
\begin{aligned}
& \frac{d}{d \theta} \frac{1+\theta^{2}}{1+\left[1 \lambda /\left(\omega-\omega_{*}\right)\right]\left(1+\theta^{2}\right)} \frac{d}{d \theta} \tilde{\phi} \\
& {\left[\frac{\omega-\omega_{*}}{\omega-(1-\varepsilon) \omega_{*}}\right] \frac{\left(1-E i D+\omega\left(\omega-E \omega_{*}\right)\left(1+\theta^{2}\right)\right.}{1+\omega b_{\theta}\left(1+\theta^{2}\right) /\left[\omega-(1-E) \omega_{*}\right]} \tilde{\phi}=0 .}
\end{aligned}
$$

We note that this equation can be also derived from the gyro-kinetic approach. 11

Although ion sound effects are neglected in Eq. (5), perpendicular conpression of plasma is representod by the $\omega^{b} \theta\left(1+\theta^{2}\right) /\left[\omega-(1-\varepsilon) \omega_{t}\right]$ term which comes from the divergence of polarization drift velocity in the pressure evolution equation. The Inertial term $\omega\left(\omega-E \omega_{*}\right)\left(1+\theta^{2}\right)$ has a factor $\omega-\varepsilon \omega$ * which makes the real frequency of the unstable mode approach $\varepsilon \omega_{\star}$ as we

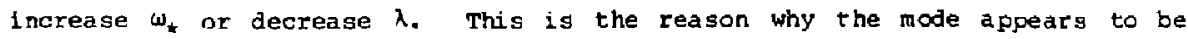
drift-wave-like.

From Eq. \{5\}, we can identify at least the following three scaling lengths. $\theta_{\lambda}{ }^{2} \equiv\left|\left(\omega-\omega_{\star}\right) / \lambda\right|$ corresponds to the inverge of resistive layer width where the stabilizing field lige bending term Ithe first term in 34. (5)) hecomes small due to reslstive dissipation at large $\theta$. By balancing the Inertia and the driving term $D(1-\varepsilon)$, we have $\theta_{D}^{2} \equiv\left|D(q-\varepsilon) / \omega\left(\omega-\varepsilon_{i}\right)\right|$. The semicollisional (finite $\rho_{s}$ ) effect becomes non-neọligible when $\theta^{2} \sim \theta_{b}^{2} \equiv$ $\left|\left[\omega-(1-\varepsilon) \omega_{\bullet}\right] / \omega_{\theta}\right|$ 
To make further analytical progress, we make the following subsidiary orderinge. First, we note that for the collisional drift-interchange mode one has $\omega \sim \mathrm{D}^{2 / 3} \lambda^{1 / 3}$ ant, thus, $\theta_{\mathrm{D}}>\theta_{\lambda}$ when $\mathrm{D}<1$. In the semicollisional regime, where $\rho_{s}$ is greater than the registive layer width, we have $\theta_{b}<\theta_{\lambda}$. As a result, we adopt the following orderings for the semicollisional driftInterchange mode

$$
\theta_{b} \ll \theta_{\lambda} \ll \theta_{D}
$$

With orderings given by Eq. (6), Eq. (5) can be approximated by the following equations in the three different asymptotic regions. In region (i), where $\theta \ll \theta_{\lambda}$, we have

$$
\frac{d}{d \theta}\left(1+\theta^{2}\right) \frac{d}{d \theta} \tilde{\phi}_{i}+\left(\frac{\omega-\omega_{\star}}{d-(1-E) \omega_{\star}}\right) \frac{(1-\varepsilon) D}{1+\omega \omega_{\theta}\left(1+\theta^{2}\right) /\left[\omega-(1-E) \omega_{\star}\right]} \tilde{\phi}_{i}=0
$$

In region (ii), where $\theta_{b} \ll \theta \ll \theta_{D}$, we have

$$
\frac{d}{d \theta} \frac{\theta^{2}}{1+1 \lambda \theta \beta\left(\omega-\omega_{\star}\right)} \frac{d}{d \theta} \tilde{\phi}_{11}+\frac{D(1-\varepsilon)\left(\omega-\omega_{\star}\right)}{b_{\theta} \omega \theta^{2}} \tilde{\phi}_{1 i}=0 .
$$

Floally, in region $(1 i t), \theta>, \theta_{\lambda}$, we have

$$
\frac{d^{2}}{d \theta^{2}} \tilde{\phi}_{i 11}+\frac{i \lambda}{b_{\theta} \omega} \frac{D(1-\varepsilon)+\omega\left(\omega-\varepsilon \omega_{n}\right) \theta^{2}}{\theta^{2}} \tilde{\phi}_{i 1 i}=0 \text {. }
$$

Wote that these three equationg have common regions of validity and, hence, we can perform asymptotic matching.

The solution of D. (9), which behaves well at large $\theta$, is given by 


$$
\widetilde{\phi}_{i i i}=\theta^{1 / 2} K_{p}\left[-i\left[\frac{i \lambda\left(\omega-\varepsilon \omega_{\star}\right]}{b_{\theta}}\right]^{1 / 2} \theta\right\} .
$$

where $K_{p}$ is the modified Bessel function, and

$$
p^{2}=\frac{1}{4}-\frac{1 \lambda D(1-E)}{{ }^{4} t_{\theta}}
$$

In Eg. (10), we require

$$
\operatorname{Im}\left[\frac{i \lambda(\omega-\varepsilon \omega}{b_{\theta}}\right]^{1 / 2}>0
$$

to ensure proper behavior as $\theta \rightarrow \infty$. In the small- $\theta$ limit. Eq. (10) leads to

$$
\frac{d \tilde{\phi}_{i j i}}{d \theta}-\theta^{-1 / 2-p}-e^{-p \pi i} \frac{1 / 2+p \Gamma(1-p)}{1 / 2-p \Gamma(1+p)}\left[\frac{i \lambda\left(\omega-\varepsilon \omega_{*}\right)}{4 b_{\theta}}\right]^{P_{\theta}-1 / 2+p} .
$$

Here, we remark that it is easier to match $d \tilde{\phi} / d \theta$ rather than $\tilde{\phi}$.

We now examine the solution of region (ii). To solve $E q$. ( $B$ ), we make the following transformation $t=1 / \theta$ and

$$
u=-\frac{\theta^{2}}{1+i \lambda \theta^{2} /\left(\omega-\omega_{*}\right)} \frac{d}{d \theta} \tilde{\phi}_{i i}=\frac{t^{2}}{t^{2}+i \lambda /\left(\omega-\omega_{\star}\right)} \frac{d}{d t} \tilde{\phi}_{i i} .
$$

Equation (B) then becomes

$$
\frac{d^{2}}{d t^{2}} U+\left[\frac{D(1-\varepsilon)\left(\omega-\omega_{2}\right)}{b_{\theta} \omega}+\frac{ \pm \lambda D(1-\varepsilon)}{\omega_{\theta} t^{2}}\right] v=0 \text {, }
$$

which has a solution

$$
u=t^{1 / 2}\left[z_{i p}(\delta t)+c_{2} z_{2 p}(\delta t)\right]
$$


where $z_{1 p}$ anc $z_{2 p}$ are Bessel functions to be specified and

$$
5^{2}=\frac{D(\theta-\varepsilon)(\omega-\omega)}{b_{\theta}^{\omega}}
$$

From Ex. (13), we have

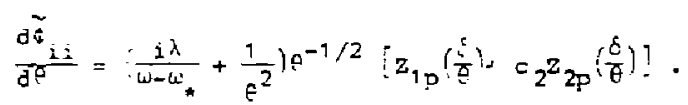

But is the reqion (ii), where 20 . (8) is valid, $\delta<s \quad \vartheta$ is satisfied such that the smail-argument Iimit of $z_{p}$ san be used in Ex. (17) both in the small- and iarge- imits of Eg. (8). Matching the large- $\theta$ solutions of Ey. (i7) with that qives by Bo. $(12)$, we have

$$
\left.z_{? p} \frac{\delta}{\theta}\right)+c_{2} z_{2 p}\left(\frac{\delta}{\theta}\right) \sim e^{-p}-e^{-p \pi i} \frac{1 / 2+p \Gamma(1-p)}{i / 2-p \Gamma(1+p)}\left[\frac{i \lambda\left(\omega-\varepsilon \omega_{-}\right)}{4 b_{\theta} p}\right]_{\theta}^{p} .
$$

Since EG. (17) is valid even in the small- $\theta$ limit of Eg. (14), we then have

$$
\begin{gathered}
\frac{d \tilde{\Phi}_{i i}}{d \theta} \sim e^{-5 / 2}\left(z_{1 p}+c_{2} z_{2 p}\right) \sim e^{-5 / 2-p}-e^{-p \pi i} \frac{(1 / 2)+p \Gamma(1-p)}{(1 / 2)-\frac{p}{p(1+p)}} \\
\times\left[\frac{i \lambda\left(\omega-\epsilon \omega_{*}\right)}{4 b}\right]_{\theta} e^{-5 / 2+p} .
\end{gathered}
$$

He now conbider the solution of Eq. (7). Since are interested in a situation where the system is stable to ideal MHD modes, suydam's criterion 12 requires $D<1 / 4$ and we can treat the second term of $B$. (7) perturbativaly. we then have

$$
\frac{d}{d \theta}\left(1+\theta^{2}\right) \frac{d}{d \theta} \tilde{\phi}_{10}=0,
$$


and

$$
\frac{d}{d \theta}\left(1+\theta^{2}\right) \frac{d}{d \sigma} \tilde{\phi}_{i 1}+\left[\frac{\omega-\omega_{\star}}{\omega-(1-\varepsilon) \omega_{\star}}\right] \frac{(1-\varepsilon) D}{1+\left[\omega \omega_{\theta}\left(1+\theta^{2}\right) /\left[\omega-(1-\varepsilon) \omega_{\star}\right]\right.} \tilde{\phi}_{i 0}=0 .
$$

Equation (20) has a solution

$$
\frac{d}{d \theta} \widetilde{\phi}_{10}=\frac{\theta_{0}}{1+\theta^{2}}
$$

where $c_{a} \rightarrow 1$ for the tearing (even- $\psi$ ) mode and $c_{0}=0$ for the twisting (odd- $\psi$ )

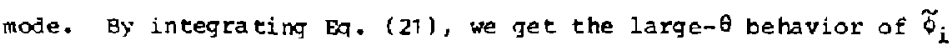

$$
\begin{aligned}
& \frac{\mathrm{d}}{\mathrm{d \theta}}\left(\tilde{\phi}_{i 0}+\tilde{\phi}_{i 1}\right) \sim\left\{\frac{-2 c_{0}}{\pi}+\frac{\pi \mathrm{D}(1-E)\left(\omega-\omega_{\star}\right)}{2 b_{\theta}^{1 / 2} \omega^{1 / 2}\left[\omega-(1-\varepsilon) \omega_{+}\right]^{1 / 2}}\right\}^{-2} \\
& -\frac{D(1-\varepsilon)(\omega-\omega)}{b_{\theta} \omega} \theta^{-3}
\end{aligned}
$$

Ey matching $9 q .(23)$ vith $\mathrm{Fq} .(19)$ and applying the $p+1 / 2$ linit since $\left|\lambda_{D}(1-\varepsilon) / \omega_{\theta}\right| \ll 1$ in the semicoilisional regime, we get

$$
\frac{\Delta^{1}}{\pi}+\frac{\pi D(1-\varepsilon)\left(\omega-\omega_{\star}\right)}{2 b_{\theta}^{1 / 2} \omega^{1 / 2}\left[\omega-(1-\varepsilon) \omega_{\star}\right]^{1 / 2}}=\frac{i^{3 / 2}\left(\omega-\omega_{\star}\right)\left(\omega-E \omega_{\star}\right)^{1 / 2}}{\left(\lambda b_{\theta}\right)^{1 / 2}},
$$

where we have replaced $-2 c_{0}$ by $\Delta^{\prime}$ according to the usual definition of $\Delta^{\prime}$ in the Fourier transformed space. ${ }^{13}$ for the modes localized around the resistive layer, one has $\Delta^{\prime}=0$ for the twisting parity and $\Delta^{\prime}=-2$ for the tearin? parity mode. Fquation (24) can also be exF ssed in the following slightly different fashion 


$$
\omega\left(\omega-\varepsilon \omega_{*}\right)\left[\omega-(1-E) \omega_{*}\right]=-1 \lambda\left\{\frac{\pi}{2} D(1-E)+\frac{\Delta b_{\theta}^{1 / 2} \omega^{1 / 2}\left[\omega-(1-\varepsilon) \omega_{*}\right]^{1 / 2}}{\pi\left(\omega-\omega_{*}\right)}\right\}^{2} .
$$

Equation (24) ox, equivalently, Eq. (25) is the desired dispersion relation for the senicollisional drift-interchange and drift-tearing modes when the perpendicular registivity and ion sound effects are neglected. In the next section, we will discuss the properties of this dispersion relation and the effects of the perpendicular resistivity and ton sound terms.

4. PROPERTIES OF DISPERSION RELATION

For localized twisting-parity maie, $\Delta=0$ and $F$. (25) becomes

$$
\omega\left(\omega-\varepsilon \omega_{\star}\right)\left[\omega-(1-\varepsilon) \omega_{\star}\right]=-i \lambda\left[\frac{\pi}{2} D(1-\varepsilon]\right]^{2} .
$$

which in the limit $E \rightarrow 0$ has the same form as shat in the collisional regime $4-6$ excent for the numerical enhanolng factor $(\pi / 2)^{2}$. Flnfte $\varepsilon$ (compression in the perpendicular direction due to curvature arift) not only reduces the effective driving term $D(1-\varepsilon)$ but also introduces a real frequency shift $\left(\omega-E \omega_{*}\right\}$. We can see that even a small $\varepsilon$ changes the behavior of the efgenfrequency aramacically as $\lambda+0$. When $\mathrm{g}=0$, we have

$$
\omega=\pi(1 \lambda)^{1 / 2} D /\left(2 w_{*}^{1 / 2}\right)
$$

as $\lambda+0$ and $4 \ll \omega_{*}$. The real frequency and growth rate are comparable and scale as $\lambda^{1 / 2}$. But, whan $E$ is finite and $\lambda \rightarrow 0$, 


$$
u=\frac{\varepsilon \omega_{\star}+i(\pi / 2)^{2} D^{2} \lambda}{E \omega_{\star}^{2}}
$$

which shows that the real frequency of the mode approaches a finite value while the growth rate becomeg very stall. This is one of the reasons why even very small perpendiculat resigtivity can atabilize the mode completely.

For the tearing-farity mode, $\Delta^{\prime}=-2$, but $\Delta^{\prime}$ ha a negligible effect on the elgenfrequency as long $a s b_{\theta}<<1$ and $\omega$ is not very clase to $\omega_{*_{e}}$ or, more precisely, when $(\pi / 2) D(1-\varepsilon) \gg\left(\Delta^{\prime} / \pi\right) \varepsilon^{1 / 2} \mathrm{~b}_{\theta}{ }^{1 / 2}$. This fact is further demonstrated in the numerical results to be discusseö in sec. 5.

It is interesting to note that, in the essentially opposite limit, i.e..

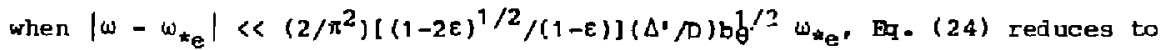

$$
\omega^{1 / 2}\left(\omega-\omega_{\star}\right)=i^{3 / 2} \frac{\Delta^{\prime}}{\pi}\left(\lambda_{b_{\theta}}\right)^{1 / 2},
$$

as $\varepsilon, n \rightarrow 0$ while keeping $b_{\theta}$ constant. Equation (29) $1 s$ the dispersion relation for the semicollisional drift-tearing mode in a sheared slab geometr $y^{7}$ in the absence of a temperature gradient. If suppose $\Delta^{\prime}>0$, Eq. (2y) shows that tike unstable branch corresponds to $\omega$ to $\omega_{k_{e}}$, and, in this case, pressure driven term, $D$, las negligible effects. Ion sound terms, on the other hand, may be important. ${ }^{8}$

Now we invegtigate the effects of perpendicular resistivity. It is more convenient to include it as a perturbation to the previous result, Eq. (24), rather than golving pq. (4) over again wth four difierent asymptotic regions. As one can see from Eq. (4), the perpendicular resistivity effect comes in only when $\theta$ is very large. For such a large value of $\theta$, Eq * (4) becones 


$$
\frac{d^{2}}{d \theta^{2}} \tilde{\phi}+i \frac{\lambda}{b_{\theta} \omega}\left[\omega\left(\omega-E \omega_{n}\right)+1 \lambda_{\perp} \frac{\Gamma \beta}{2} \omega \theta^{2}\right] \tilde{\phi}=0 .
$$

The unperturbed equation (zeroth order in $\lambda_{\perp}$ ) is given by

$$
\frac{a^{2}}{d \theta^{2}} \tilde{\phi}^{0}+1 \frac{\lambda}{b_{\theta}}\left(\omega_{0}-\epsilon \omega_{\star}\right) \tilde{\phi}^{0}=0
$$

By keeping in 5 . $(30)$ terms of first order in $\lambda_{1}$, we have

$$
\frac{d^{2}}{d e^{2}} \tilde{\phi}^{1}+\frac{i \lambda}{b_{\theta}}\left(\omega_{0}-\varepsilon \omega_{*}\right) \tilde{\phi}^{1}+\frac{i \lambda}{b_{\theta}}\left(\omega_{1}+i \lambda_{\perp} \frac{\Gamma \beta}{2} \theta^{2}\right) \tilde{\phi}^{0}=0 .
$$

Imposing the solubility condition, we have

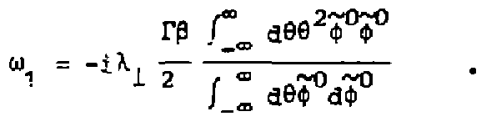

Since most of the contribution to the integral cones from the large- $\theta$ region where $\tilde{\phi}^{0}=\exp \left(-\left[-i \lambda\left(\omega_{0}-E \omega_{\star}\right) / b_{\theta}\right]^{\dagger / 2} \theta\right\}$, we have

$$
\omega_{1} \cdot \frac{\lambda_{1}}{4 \lambda} \frac{\Gamma \beta b_{\theta}}{\left(\omega_{0}-\varepsilon \omega_{*}\right)} \text {. }
$$

$\omega_{1}$ introduces a stabilizing effect since $\operatorname{Re}\left(\omega_{0}-\varepsilon \omega_{*}\right)>0$ and $\operatorname{Im} \omega_{0}>C$. Physically, the perpendicular transport of plasma smears out the pressure perturbations near the resistive layer and, hence, provides stabilization. Equation (25) wh th. (34) gives the eigenfrequency for the senicollistonal Arift-interchange and drift-tearing nodes. For the drift-interchange modes, we can obtain the following aritical value of $b_{*}$ above which the mote is stable by balancing the approximate growth rate given by . . (28) against the damping rate given by $\omega_{1}$ of Eq. (34): i.e., 


$$
\omega_{*_{C}}=\left(\frac{\pi^{2}}{2}\right)^{1 / 3}\left(\frac{\lambda}{\lambda_{\perp}}\right)^{1 / 6} \frac{\lambda^{1 / 3} D^{5 / 6}}{e^{5 / 2}\left(\Gamma^{1}\right)^{1 / 6}}
$$

or, in terms of $b_{\theta}$.

$$
b_{\theta_{c}}=\left(\frac{\pi^{2}}{2}\right)^{2 / 2}\left(\frac{\lambda}{\lambda_{1}}\right)^{1 / 3} \frac{\lambda^{2 / 3} D^{2 / 3}}{(\Gamma \beta)^{1 / 3}}
$$

Note that both finite- $\beta$ (compreggion) and the perpendicular resistivity are necessary for copplete stabilization. Bowever, for the drift-tearing rode,

$$
\omega_{0}=\omega_{*}+i^{3 / 2} \frac{\Delta^{1}}{\pi} \frac{\left(\lambda b_{\theta}\right)^{7 / 2}}{\omega_{\star}^{i / 2}}
$$

and, hence, the damplng rate avallable fron $\omega_{1}$ is always smaller than the growth rate given by $\omega_{0}$ by the ratio $\lambda_{\perp} \varepsilon \Gamma \beta / 4 \lambda D$, and the effect of perpendicular regigtivity ig negligible.

Now we can show that the ion sound term is actualy negligible for the semicollisional drift-interchange gode. To denonstrate it, we take the ratio of two representative terms from ㅂ. (3) and find

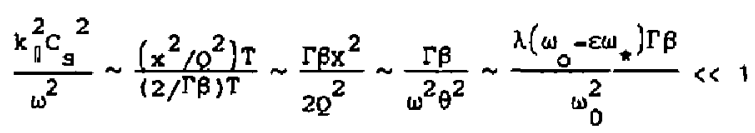

based on the senlcollisional ordering and $\theta$ ls taken to the typical mode widh. Therefore, the fon sound term becomes negligible as we increase $b_{\theta}$ or decrease $\lambda$, On the other hand, $k_{0}^{2} c_{g}^{2} / \omega^{2} \sim \Gamma \beta / 2 D$ in the collisional regine and Ion sound teriss carnot always be Ignored. 
16

5. SUMERICAL RESUTS

In this section, we describe the results obtained by integrating numerir.sty the full set of equations; Eq. (1), (2), and (3). The specific purpose is to resolve the differences between our conclusion and that of FMA's on the main stabilizing mechanism for the restorative interchange modes. As mentioned in sec. 2, EMs. (1), (2), and (3) can be Fourier transformed into the following fourth order system

$$
\frac{a}{d \mu} \frac{\mu^{2}}{1+\mu^{2},\left(\phi+i Q_{\star}\right)} \frac{d}{d \mu} \ddot{\phi}+\left(0-Q^{2} \mu^{2}\right) \tilde{\phi}-\left(D+i \phi \rho_{\star} \mu^{2}\right) \tilde{\xi}=0 \text {. }
$$

and

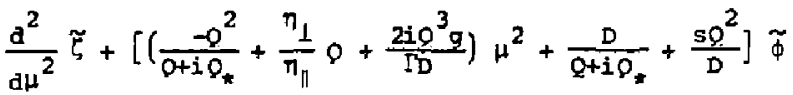

$$
\begin{aligned}
& -\left(\frac{1 Q Q_{\star}}{Q+i Q_{\star}}+\frac{\eta_{1}}{\eta_{\|}} \rho+\frac{2 Q^{2} Q_{\star} g}{\Gamma D}\right) \mu^{2} \xi \\
& +\left[\frac{D}{Q+i Q_{\star}}+\left[1+\frac{2}{\Gamma \beta}\right) Q\left(Q+i \rho_{\star}\right)-\frac{i \phi_{*}^{s}}{D}\right] \xi=0
\end{aligned}
$$

where

$$
\begin{aligned}
& \tilde{\phi}(\mu)=\int_{-\infty}^{\infty} \mathrm{dxe} e^{i x \mu} \phi(x), \\
& \varphi(x)=D E(x)+\frac{j \varphi_{2}}{Q} T(x),
\end{aligned}
$$

$a \pi d$

$$
\zeta(x)=D \Xi(x)-T(x)
$$


Equation (3B) is apparently singular at $\mu=0$. Thlo singilarity can be removed by including a $k_{\theta}^{2}$ contribution to $\nabla_{\perp}^{2}$. S'nce length has been already normalized wi th respect to the resistivity in Eqg. (1), (2), and (3) according to the so-called slow intarchange ordering, ${ }^{2}$ it 18 rather awkward to express $\dot{\kappa}_{\theta}^{2}$ in FMA's notation. From now on, we we the notation defined in Eq. (4). Equations (37) and (38) can then be writtel as

$$
\begin{aligned}
& \frac{d}{d \theta} \frac{1+\theta^{2}}{1+\left[i \lambda /\left(\omega-\omega_{\star}\right)\right]\left(1+\theta^{2}\right)} \frac{d}{d \theta} \tilde{\phi}+\left[D+\omega^{2}\left(1+\theta^{2}\right)\right] \tilde{\phi}-\left[D+\omega \omega_{*}\left(1+\theta^{2}\right)\right] \tilde{\zeta}=0, \\
& \frac{d^{2}}{d \theta^{2}} \tilde{\zeta}-\left[\left(\frac{-i \lambda \omega^{2}}{\omega-\omega_{\star}}+i \lambda_{\perp} \omega+\frac{s \omega_{\star} \omega^{3}}{\Gamma_{D}^{2}}\right)\left(1+\theta^{2}\right)+\frac{i \lambda D}{\omega-\alpha \omega_{*}}+\frac{g \omega^{2}}{D}\right] \tilde{\phi} \\
& \quad+\left(\frac{-i \lambda \omega \omega_{\star}}{\omega-\omega_{\star}}+i \lambda_{\perp} \omega+\frac{5 \omega^{2} \omega_{\star}^{2}}{\Gamma D^{2}}\right)\left(1+\theta^{2}\right) \tilde{\zeta} \\
& \quad+\left[\frac{-i \lambda D}{\omega-\omega_{\star}}+\left(1+\frac{2}{\Gamma \beta}\right) \omega\left(\omega-\omega_{\star}\right)+\frac{\omega \omega_{\star} s}{D}\right] \zeta^{2}=0 .
\end{aligned}
$$

Equations (39) and (40) are the basic equations for our numerical calculations. We again note that our model equation for the analytical calculation, Eq. (4), can be derlved from Eqs. (39) and (40) by neglecting the ion sound terms.

Elgenfrequencies are found using the shooting method for both the fourth order system, Eqs. (39) and (40), ant the second order system Bq. (4). The parameters are chosen to be relevant to current Reversed Fleid Pinch experiments ${ }^{3}$ as in the work of FMA; and, therefore, direct comparison with their results is possible. In particular, effects of the perpendicular resistivity and fon sound wave are examined. To isolate the effects of the perpendiculax residilivity, we examine two cases with $\lambda_{1} / \lambda=0$ and $\lambda_{1} / \lambda=1$, 
respectively. For each case, we integrate both the full set of equations wth Ion sound terms, Eqs, (39) and (40), and che mojlel equation without lon sound terms, Dr. (4). The obtained complex eigenmode friquencles are plotted in Figs. (i) to (4) as functions of $\omega_{*}$; while keeping the other parameters $0, \beta$, $\Delta \cdot s, \lambda, \lambda_{1}$ fixed.

In F1gE. (1) and (2) where the perpendicular resigtivity is set to zero, we do not observe the complete stabilization of the modes no matter how large $\omega_{*}$ ig increased. This conclusion is the same whether we include fon sound terms or not. The ion sound effects, in fact, are negligible in the semicollisional regime as we have Indicated in Sec. 4. We also have very good agreenent between analyttcal [Eq. (25)] and numerical results in the semicollisional regime.

On the other hand, in Figg. (3) and (4) where the perpendicular resistivity is kept finite, we observe the complete stabilization of the rodes for the moderate value of $\omega_{\boldsymbol{s}_{e}}$ whether ton sound terms are kept or not. In this case, again, the effects of ion sound waves are negliqible and the agreements between analytical [Eqs. (25), (34) and (75)] and numerical results are very good in the semicollistonal regime.

We also note that the behavior of the elgenfrequencies are almost the same for the even and odd parity modes because, for the pressure driven modes we are considering, the effect of the ldeal MHD region (characterized by $\Delta^{\prime}$ ) Is small as we have noted in Sec, 4.

summarizing tiss section, we have numerically found that the semicollisional drift-interchange modes can be completely stabllized for a moderate value of $\omega_{w_{e}}$ as indicated in FMA. We, however, have shown that the maln stabilizing effect is due to perperdicular transport of the plasma. The Ion sound terms, claimed as the main stabilizing mechanism in FMA, meanwhila, 
have negligible effects on the mode frequencles and gtabilitles.

\title{
6. CONCLUSIONS AND DISCUSSIONS
}

\begin{abstract}
We have studied the semicollisional drift-interchange modes in a cylinarical geometry. It is shown, both analytically and numerically, that the modes can be completely gtabllized for moderate ralue of $\omega_{\mathrm{e}^{*}}$ It is, furthermore, clearly demonstrated that perpenilcular transport of the plasma is the main stabilization rechanism while ion sound effects axe negligible. This is because due to the small parallel resistivity compared to the collisional cage, the mode is more locallzed near the singular surface and perpendicular transport of plasma can readily smear out the instabilitydriving pressure perturbations in that narrow region, This has been explicitly shown in sec. 4. The damplng rate induced by the plagma transport [ classical plasma diffusion coefficient/(mode widthic] is given by Eq. (34). By balancing tha damping rate againgt the qrowth rate given by Eq. (2g), we are also able to estimate the critical value of $\omega_{*}, \omega_{* c}$ [Eq. (35)], at which the mode becomes stabilized, and the results agree with the numerical results excellenty.
\end{abstract}

\section{ACKROWLEDQMENTS}

This work is supported by the Unlted States Departuent of Energy Contract No. DE-ACO2-76-CHO-3073. The authorg would like to thank H. S. Chance, S. C. Jardin, พ. M. Tang, J. พ. Connor, and G. W. Hammett for useful discussions. 
REF ERENCES

1H. Y. Furth, J. Killeen, and M. N. Rosenbluth, Phys. Pluids E, 459 (1963).

2B. coppl, J. M. Greene, and J. I. Johnson, Mucl. Fusion 6 , 101 (1966).

$3_{z T-40}$ Iear, in Proceed Ings of the N1nth International conference on Placma Physics and Controlled Fusion Research, Baltimore, Maryland, 1982, IA EA $-\mathrm{NN}-47 / \mathrm{H}-2$.

4. Coppi, Phys. Muids I, 1501 (1964).

5z. G. An and p. f. Diamond, in proceedings of the Sherwood Theory Meeting, arlington, Vizginia, 1983, Faper 2523.

${ }^{6}$ G. w. Hamnett and W. H. Tang, Nucl. Fusion 23, 1503 (7983).

${ }^{7}$ J. F. Drake and Y. C. Lee, Fhya. Flulds 20, 1341 (1977).

g. N. Bussac, D. Edery, R. Pellat, and J. I. Soule, Phys. Rev. Lett. 40, $1500(1979)$.

9. M. Finn, H. N. Manheimer, and T. M. Antonsen, Phys. Fluids 26,962 (1983).

10J. w. connor, W. M. Tang, and J. B. Taylor, Phys. Fluids 26, 158 (1303).

11 T. S. Hah, Ph. D. Theg1s, Princeton University (9984).

${ }^{12}$ B. R. Suydam, In Proceedings of the U.s. International Conference on Peaceful Uses of Atonic Bnergy, Geneva (Colubia d. P., New York, 1959) Vol. 31. p. 157.

13H. R. Straugs, Phys. Fluids 24, 2004 (1982). 
FIGURE CAPTIONS

FIG. 1 Plot of twigting-parity eigermode freoriersieg versis $\omega_{\star_{e}}$ The parameters are $\lambda=10^{-6}, D=0.14, \Gamma \beta=0.0512, s=0.7, \lambda_{1}=0$, and $\Delta^{\prime}=0$. $x$ cciresponds to aralytical results given by Eqs. (25) and (34). 0 and 4 and are direct numerical regults with (Eqs. (39) and (40)] and without [Eq. (4)] ion-sound effects.

FIG. 2. Plot of tearing-parity eigennode frequencies versus $\omega_{e^{*}}$ The parameters are $\lambda=10^{-6}, D=0.14, \Gamma \beta=0.0512,3=* .7, \lambda_{\perp}=0$, and $\Delta^{*}=-2$. $x$ corresponds to analytical results given by Egs. (25) and (31). 0 and 4 and are direct numerical regults with [Egs. (39) and (40)! and without [Eq. (4)] ion-sound effects.

FIG. 3. Plot of twisting-parity eigemode frequencies versus $\omega_{k_{e}}$. The parameters are $\lambda=10^{-6}, D=0.14, \Gamma \beta=0.0512, s=0.7$, and $\Delta^{\prime}=0$. $x$ corregponds to analytical results given by Eqs, (25) and (34). 0 and and are direct numerical results with [Eqs. (39) and (40)] and without [Eg. (4)] ion-gound effects.

FIG. 4. Plot of tearing-parity eigenmode frequencieg vergus $\omega^{\omega_{e}}$. The parameters are $\lambda=10^{-6}, D=0.14, \Gamma \theta=0.0512, \mathrm{~s}=0.7$, and $\Delta^{\prime}=$ -2. $x$ corresponds to analytical resultg given by Eqs. (25) and (34). O and $\Delta$ are direct numerical regults with [Eqs. (39) and (40)] and without [Eq. (4)] ion-gound effects. 

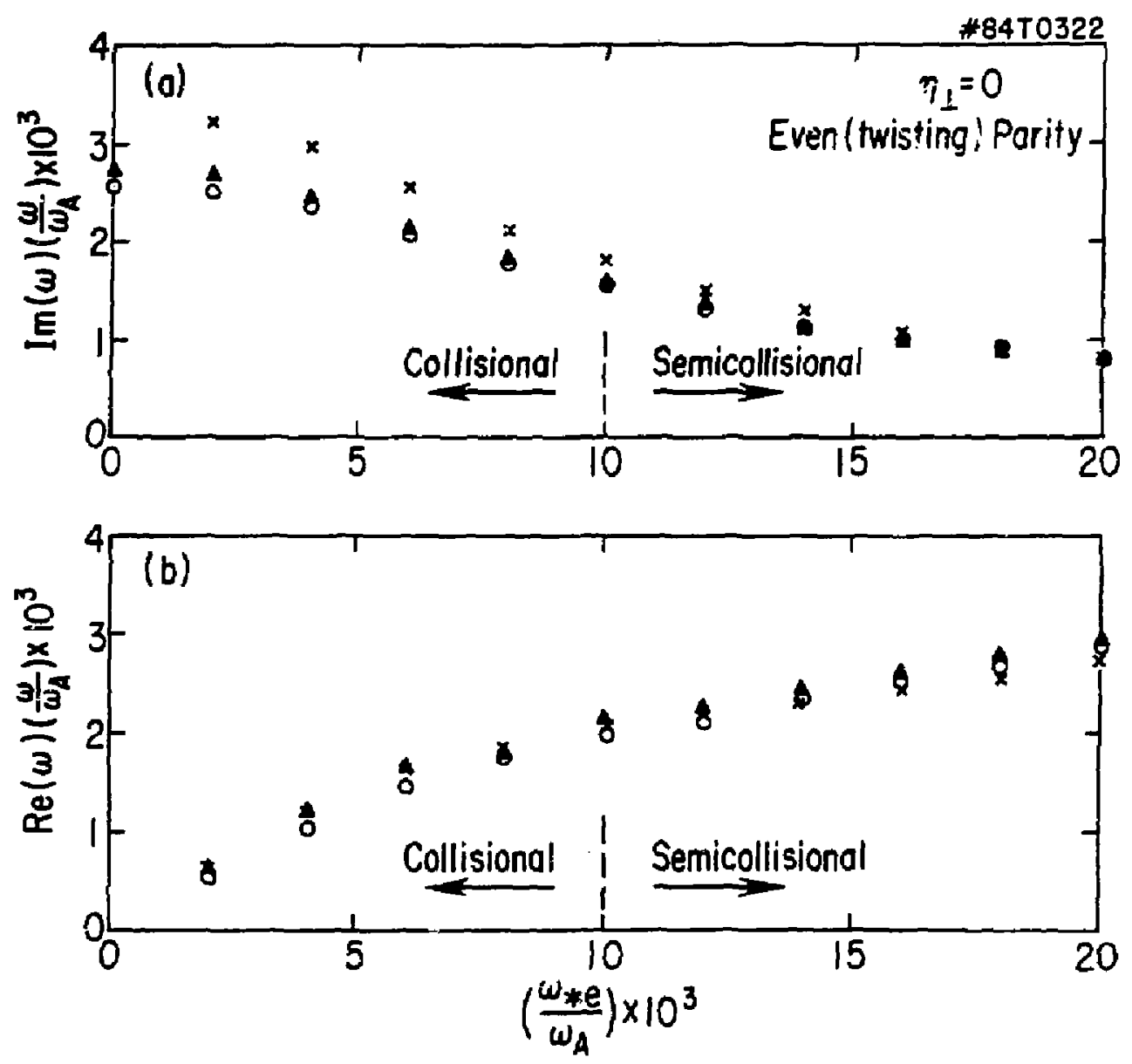

Fig, 1 

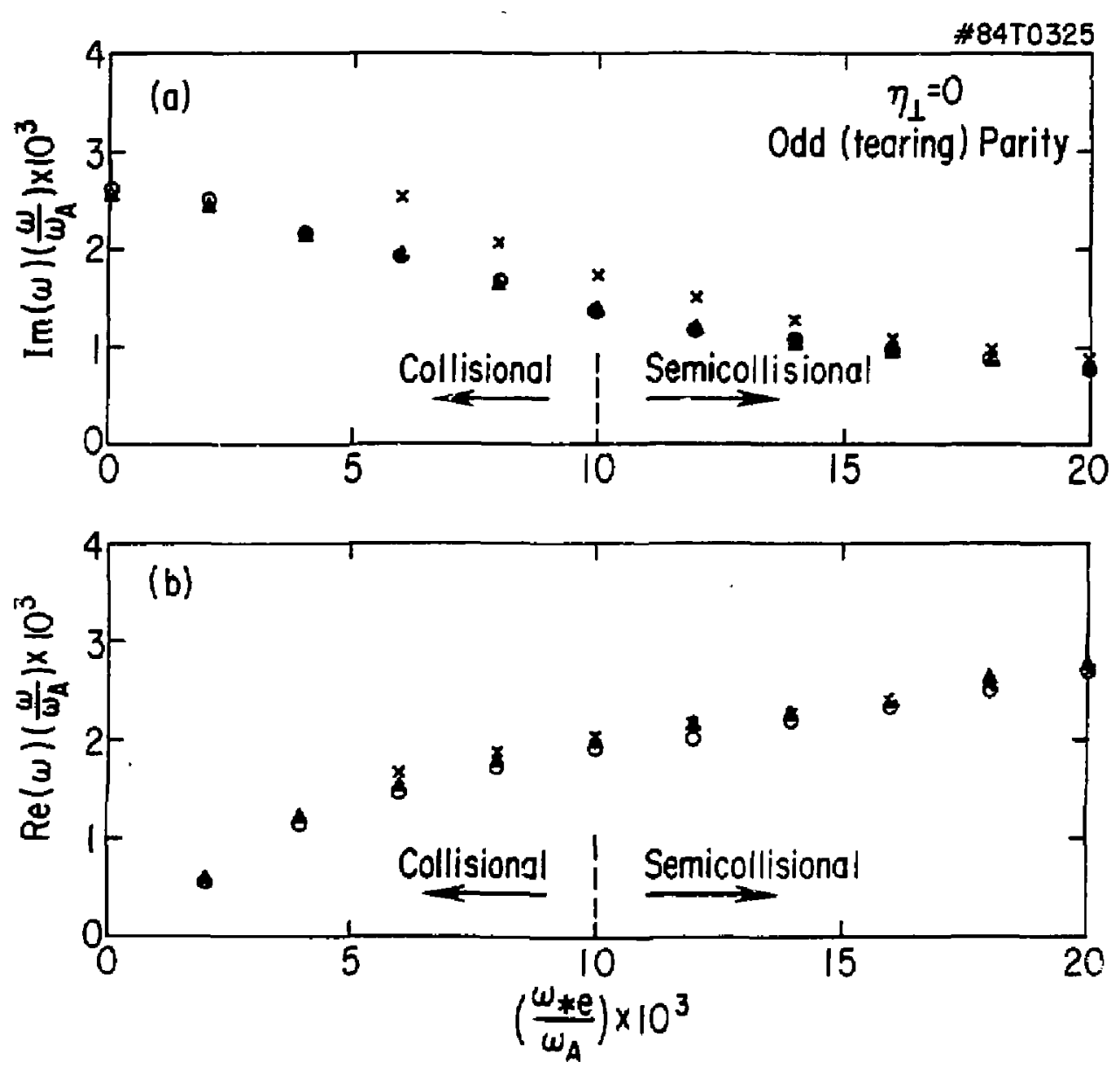

Fig. 2 

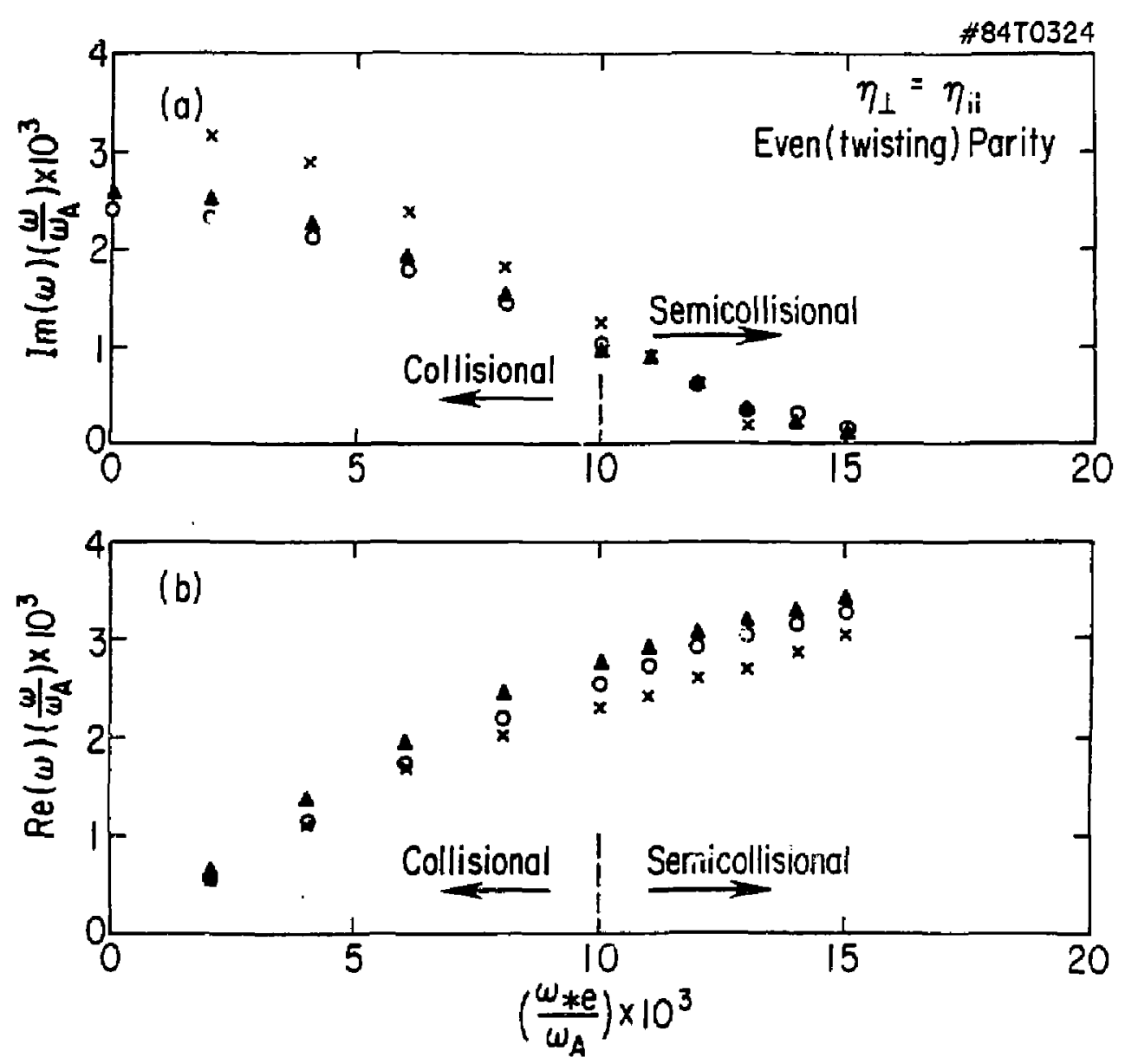

Fig. 3 

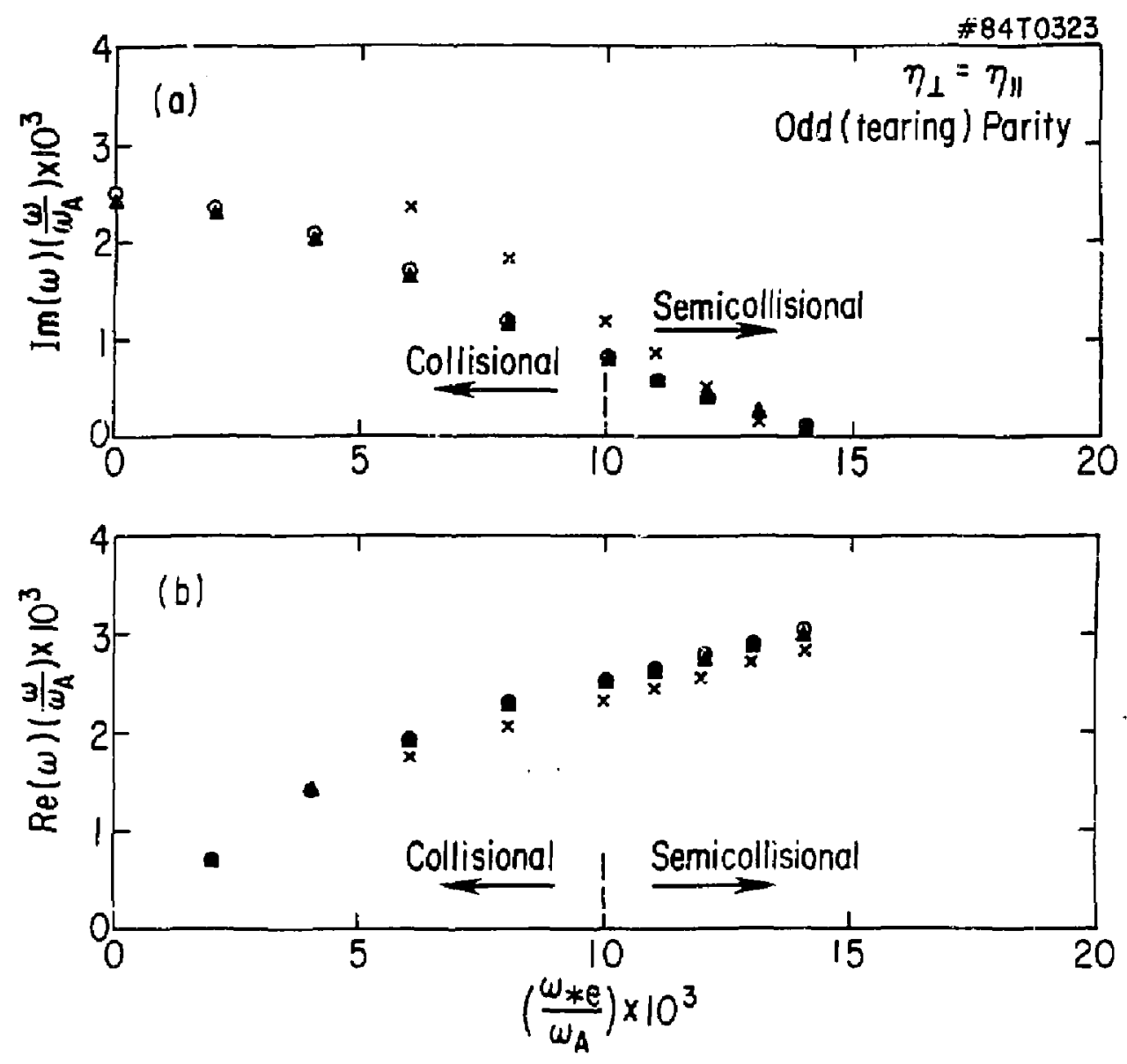

Fig. 4 
Plasra Res Lab, Augta kat'l Univ, Astranta Dx. Ftark J. Eaoloni, thiy of Kollongong, ALSTRALTA Erof. I.R Janes, Flindarg Inive, ALSIRALIA Prof. M.H. Beennar, thiv Sydney, AUSLRALIA Prof. F. Cap, Ingt Theo Fhys, Austrita prof. Lrark Verteost, Inst theoretische, BareIIM Dr. D. Palúbo, og XII Asaim Prog, BFIGTCM Ecole Royale Militalre, Lab de Fhys Plamas, BEIGIUM Dr. P.H. Sakanalka, Univ Estaduel, ERATI Dr. C.R. James, Oniv of Alberta, CANFA Prof. J. Teirtmann, Univ of Montreal, CARDA DT. H.M. Skaygart, Univ of Sagkatheran, CANDA Frof, 5.R. Speentuasan, University of Calgary, CARDA ExoE. Tudor W. Johnston, INFS-phergie, CHFA DT. Handes Bamant, Driv British Columbia, CNALA Dr. M.P. Bachynski, MPB Techrologied, Ine, CARPAA Chalk River, Mucl Iab, OKanA

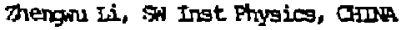

Tibrary, Tsing tha University, CHN Uibrarian, Instutute of Ihysics, Cemp Inst Plasta Finys, Acadenia SInica, Gink 7I. Peter Lukac, Kanengkeho Univ, Curchsionakn The Librarian, Culfaru Labsratory, ExCAND Prof, Schatzman, obserataire de Nice, FRANCE J. Radet, COR-BE, RRANE AM Dupas Library, AM Dujas Iibrary, BRALCE DI. Tum Mal, Acadeny PAbliographic, tovg kDo Preprint Library, Cent Res Inst Phys, ftMkeafy DT. S.K. Trehan, Panjah University, INDIA Dr. Indra tohan Lal Das, Banares findu Univ, nDTa Or. I.K. Sravia, South Gujarat Univ, INDIA Dr. R.K. Chhajlani, Vikram thiv. INDIA D.. 3. Dasgupta, Sala Inst, IDIA Dr. P. Kas, Rtysical Research Iab, INDIA Dr. Phillip Rosenau, Istael Inst Tech, tsaAar Prof. s. Copeman, Tel Aviv Oniversity, ISFAEI. Fruf. G. Rostagni, thit Di Padorn, IIALY Iibrariar, Int' 1 Ctr theo Phys, IXWY

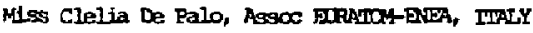
Biblioteca, del CNR ERATOA, IIFLY DE. H. Yamato, Tositiba Reg \& Dev, JAFAN Direa Dept Eg. Tokanak Dev. MALRI, JAPAN Prof. Hoturyiki Inave, University of Takyo, JARAN pesearch Info Center, Nagoya thiversity, JAPAN Prof. Kyoji Nishikasa, Oniv of Hiroahima, JAPAN ProI. Sigenu HoEl, JAERI, JAFAN Librarg, Kyoto universit ty, Jagrol Prof. Ichiro Kanakan, Whon Driv, JAPAN Prof. Satrahn Itah, Kyushe Oniyersity, JApaN DF. D.I. Ohai, Atv. Inst Sei \& Tech, KORER Tech Info Divigion, KARRI, KDREA Bibliothrek, Fam-Inst voor Plasma, NEIHGLANDS
Erof. E.5. LLley, University of Waikato, NEN ZFALAND Prof. J.A.C. Cabtal, Inte Supecior Tean, PORTucal Dr. Octavian Petrus, ALI OZA University, ROMANIA Prof. M.A. Hellberg, Universtty of Natal, SO AFRICA DT. Johan de villiers, Plasma Physict, Nucor, so AFRICA Fusion O14. LAbrary, JN, SPAI

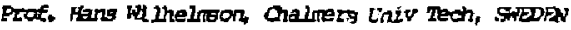
Dr. Lemart Stenflo, Uniluersity of UMEA, SWEDEN Eibrary, Boyral Inst Tech, SHEDON

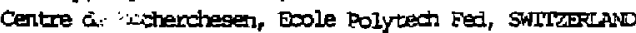
De. V.T. Tolok, Khapkov Ftryg Tech IIs, ISSR Dc. D.D, Ryutor, Slberian Acal Sci, USSR Dr. G.A. Elifev, Kurchatov Institute, ISSR Dr. V.A. Glvikhikh, Inst Electro-Physical, USSR Irstitute Ger. Ftysion, USSR Prof. T.J.M. Byrd, Univ college w wales, WALES DT. Ko Schinaler, Fuhe Universitat, t. GrapaNy Nuclear Res Bsteb, Julich Itd, W. CeRMaN Librarian, Max-Plandk Institat, w. GRRMNY Bibliothek. Inst Elasmaforschung, w. GBFANY Prof. R.K. Janev, Inst Phys, YuecestavIa 